\title{
SOME COUNTABILITY CONDITIONS ON COMMUTATIVE RING EXTENSIONS
}

\author{
BY \\ ROBERT GILMER $^{1}$ AND WILLIAM HEINZER ${ }^{2}$
}

\begin{abstract}
If $S$ is a finitely generated unitary extension ring of the commutative ring $R$, then $S$ cannot be expressed as the union of a strictly ascending sequence $\left\{R_{n}\right\}_{n=1}^{\infty}$ of intermediate subrings. A primary concern of this paper is that of determining the class of commutative rings $T$ for which the converse holds-that is, each unitary extension of $T$ not expressible as $\cup_{1}^{\infty} T_{i}$ is finitely generated over $T$.
\end{abstract}

1. Introduction. In [1], the notions of $\left({ }^{*}\right)$ - and $\left({ }^{* *}\right)$-modules are introduced in connection with the problem of determining whether a commutative ring $R$ is Noetherian if $P[[X]]=P \cdot R[[X]]$ (power series ring extension) for each prime ideal $P$ of $R$. The definitions are as follows. The unitary module $M$ over the commutative ring $R$ is a (*)-module if each countably generated submodule of $M$ is contained in a finitely generated submodule of $M, M$ is a ( $\left.{ }^{* *}\right)$-module if $M$ cannot be expressed as the union of a strictly ascending sequence of submodules. In general,

$$
M \text { finitely generated } \Rightarrow\left({ }^{*}\right) \Rightarrow\left({ }^{* *}\right),
$$

and neither of the reverse implications holds. But it is shown in [1] that the class $\mathscr{F}$ of rings over which each $\left({ }^{* *}\right)$-module is finitely generated includes the subclasses of Noetherian rings, finite-dimensional chained rings, and $W^{*}$-rings.

In this paper, we investigate the analogues of conditions $\left({ }^{*}\right)$ and $\left({ }^{* *}\right)$ for ring extensions. Specifically, if $R$ is a commutative ring with identity and $S$ is a commutative unitary extension ring of $R$, then we say that $S$ is a (*)-extension of $R$ if each countably generated extension $R\left[\left\{s_{i}\right\}_{1}^{\infty}\right]$ of $R$ in $S$ is contained in a finitely generated extension of $R$ in $S$; $S$ is a ( $\left.{ }^{* *}\right)$-extension of $R$ if $S$ is not of the form $\cup_{i=1}^{\infty} S_{i}$, where $\left\{S_{i}\right\}_{i=1}^{\infty}$ is a strictly ascending sequence of subrings of $S$ containing $R$. Again $S$ finitely generated over $R \Rightarrow S$ is a $\left(^{*}\right)$-extension $\Rightarrow S$ is a (**)-extension, and neither of the reverse implications holds in the general case. The problem of determining the classes of rings for which each (**)-extension (or (*)-extension) is finitely generated is the central focus of this paper.

After proving some preliminary results in $\$ 2$, we prove in $\$ 3$ that if the integral domain $J$ is a $\left(^{* *}\right)$-extension of its subring $D$, where $D$ has Noetherian spectrum,

Received by the editors February 29, 1980.

1980 Mathematics Subject Classification. Primary 13B02, 13E15; Secondary 13E05, 13G05, 16A30.

Key words and phrases. Finitely generated ring extension, Noetherian spectrum, countably generated ideal, von Neumann regular ring.

${ }^{1}$ Research supported by NSF Grant 7903123.

${ }^{2}$ Research supported by NSF Grant 78-00798. 
satisfies d.c.c. for prime ideals, and has each of its ideals countably generated, then $J$ is a finitely generated extension of $D$ (Theorem 3.7); in $\$ 5$, this result is extended in Theorem 5.3 to show that an arbitrary $\left(^{*}\right)$-extension of a ring satisfying the same three conditions is a finitely generated extension. Theorem 3.10 is the result that a (**)-extension $T$ of a Noetherian ring $R$ is finitely generated over $R$ if and only if $T$ is Noetherian.

Let $k$ be a field, let $S$ be the direct product of $k$ with itself over an infinite index set $N$, and let $k^{*}$ be the diagonal imbedding of $k$ in $S$. In $\$ 4$ we observe that $S$ is not a $\left({ }^{*}\right)$-extension of the field $k^{*}$, and that $S$ is a $\left({ }^{* *}\right)$-extension of $k^{*}$ if and only if $k$ is finite (Proposition 4.3 and Theorem 4.7). We are indebted to Leonard Lipschitz for the result that for $k$ finite, $S$ is a $\left({ }^{* *}\right)$-extension of $k^{*}$ that is not finitely generated. This result shows that there are some fairly stringent limitations on possible extensions of the results of $\S 3$.

Finally, in $\S 6$, we define $\mathfrak{T}$ to be the class of rings $R$ such that $R$ has Noetherian spectrum, d.c.c. for prime ideals holds in $R$, and either (3) each ideal of $R$ is countably generated, or (4) each ideal of $R$ contains a power of its radical. We prove in Theorem 6.1 that $\mathscr{T}$ is contained in the class $\mathscr{F}$, defined in [1], consisting of rings over which each $\left({ }^{* *}\right)$-module is finitely generated. Noting that $\mathfrak{N}$ properly contains the union of the classes of Noetherian rings, finite-dimensional chained rings, and $W^{*}$-rings, it follows that Theorem 6.1 represents an actual expansion of $\mathscr{F}$ as known from [1].

All rings considered are assumed to be commutative rings with identity. If $R$ is a subring of $S$, we assume that $R$ and $S$ have the same identity element, which is the meaning of the word unitary in the statement that $S$ is a unitary extension ring of $R$; a subring $T$ of $S$ containing $R$ is referred to as an $S$-overring of $R$, and the term overring of $R$ refers to an $S$-overring, where $S$ is the total quotient ring of $R$.

2. Preliminaries. In this section, we list some results concerning conditions $\left(^{*}\right)$ and $\left({ }^{* *}\right)$ that are used frequently in the rest of the paper. Also, by examining the case of overrrings of an integral domain, we show that no pair of the conditions (*), $\left({ }^{* *}\right)$, and finite generation is equivalent.

Proposition 2.1. Consider the following conditions on a unitary extension $S$ of a commutative ring $R$.

(1) $S$ is finitely generated over $R$.

(2) $S$ is $a\left(^{*}\right)$-extension of $R$.

(3) $S$ is a $\left(^{* *}\right)$-extension of $R$.

Then (1) implies (2) and (2) implies (3).

Proof. That (1) implies (2) is patent. If (3) fails and if $S$ is the union of the strictly ascending sequence $\left\{R_{i}\right\}_{i=1}^{\infty}$ of $S$-overrings of $R$, then choose $s_{i} \in R_{i+1}-$ $R_{i}$ for each $i$. The ring $R\left[\left\{s_{i}\right\}^{\infty}\right]$ is contained in no $R_{i}$, and hence in no finitely generated extension of $R$ in $S$; that is, (2) fails if (3) fails.

The proof of the next result is standard, and is therefore omitted. 
Proposition 2.2. Let $S$ be a unitary extension ring of $R$, let $T$ be an $S$-overring of $R$, let $U$ be a multiplicative system in $R$, and let $A$ be an ideal of $S$.

(1) If $S$ is a (*)-extension of $R$, then $S$ is a (*)-extension of $T, U^{-1} S$ is a $\left(^{*}\right)$-extension of $U^{-1} R$, and $S / A$ is a $\left(^{*}\right)$-extension of $R /(A \cap R)$.

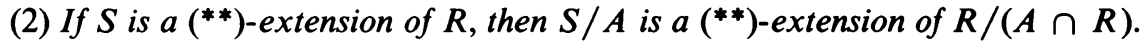

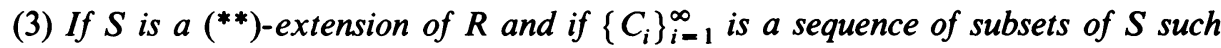
that $S=R\left[\cup_{1}^{\infty} C_{i}\right]$, then $S=R\left[\cup_{i=1}^{n} C_{i}\right]$ for some $n$; in particular, if $S$ is countably generated over $R$, then $S$ is finitely generated over $R$.

Let $D$ be an integral domain with quotient field $K$ and let $J$ be an overring of $D$. We consider briefly in the remainder of this section the problem of determining conditions under which $K$ (or $J$ ) is a $\left(^{*}\right)$ - or $\left(^{* *}\right)$-extension of $D$. Even in this context we see that $K$ a $\left(^{*}\right)$-extension of $D$ need not imply that $K$ is finitely generated over $D$; in $\S 4$ we give an example of a $\left(^{* *}\right)$-overring $J$ of a domain $D$ such that $J$ is not a $\left(^{*}\right)$-overring of $D$.

We say that a family $\left\{D_{\alpha}\right\}$ of overrings of $D$ is of finite character if each element of $K$ belongs to all but a finite number of the domains $D_{\alpha}$; the domain $D$ is of finite character if there exists a family $\left\{V_{\alpha}\right\}$ of valuation overrings of $D$ of finite character such that $D=\cap_{\alpha} V_{\alpha}$, and in this case, $\left\{V_{\alpha}\right\}$ is called a defining family for $D$. Thus, a Krull domain is a domain of finite character in which each $V_{\alpha}$ can be taken to be discrete of rank one $[4, \S 43]$.

Proposition 2.3. Let $\left\{D_{i}\right\}_{i=1}^{\infty}$ be a family of overrings of the domain $D$ such that $\left\{D_{i}\right\}_{1}^{\infty}$ has finite character. If $J$ is an overring of $D$ that is $a\left({ }^{* *}\right)$-extension of $D$, then $J$ is contained in all but finitely many of the domains $D_{i}$.

Proof. Let $J_{n}=\bigcap_{i=n}^{\infty} D_{i}$ for each $n$. Then $D \subseteq J_{1} \subseteq J_{2} \subseteq \cdots$, and since $\left\{D_{i}\right\}$ has finite character, then $K=\cup_{i=1}^{\infty} J_{i}$. Thus $J=\cup_{1}^{\infty}\left(J \cap J_{i}\right)$, which means $J=J \cap J_{k}$ for some $k$, and hence $J \subseteq D_{i}$ for each $i>k$.

Proposition 2.4. Assume that $J$ is an overring of the domain $D$ and that $N$ is a multiplicative system in $D$.

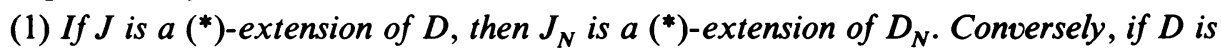
semi-quasi-local with maximal ideals $M_{1}, M_{2}, \ldots, M_{n}$ and if $J_{D-M_{i}}$ is a (*)-extension of $D_{M_{i}}$ for each $i$, then $J$ is a $\left(^{*}\right)$-extension of $D$.

(2) The analogue of (1) for $\left({ }^{* *}\right)$ is also valid.

Again the straightforward verification of Proposition 2.4 is omitted. The second statement in (1) does not generalize to the case where $D$ has infinitely many maximal ideals. For example, the rational field $Q$ is not a $\left({ }^{* *}\right)$-extension of $Z$, but $Q$ is a simple extension of $Z_{p Z}$ for each prime $p$.

Proposition 2.5. Assume that $D$ is a domain of finite character with quotient field $K$. Then $K$ is a (**)-extension of $D$ if and only if $D$ is a semi-quasi-local Bezout domain such that $K$ is a $\left(^{* *}\right)$-extension of $D_{M}$ for each maximal ideal $M$ of $D$.

Proof. It follows from Proposition 2.4 that the stated conditions are sufficient in order that $K$ should be a $\left(^{* *}\right)$-extension of $D$. Conversely, if $K$ is a ( $\left.{ }^{* *}\right)$-extension of 
$D$ and if $\left\{V_{\alpha}\right\}$ is a defining family for $D$, then Proposition 2.3 implies that $\left\{V_{\alpha}\right\}$ is finite. Thus, $D$ is a finite intersection of valuation rings on $K$, hence a semi-quasilocal Bezout domain [9, (11.11)], [4, (7.4)], and Proposition 2.4 shows that $K$ is a $\left({ }^{* *}\right)$-extension of $D_{M}$ for each maximal ideal $M$ of $D$.

If $D$ is a domain with quotient field $K$ and with integral closure $\bar{D}$, then it is true in general that $K$ is a $\left(^{* *}\right)$-extension of $D$ if and only if $K$ is a $\left(^{* *}\right)$-extension of $\bar{D}$. If $D$ is Noetherian, then $\bar{D}$ is a Krull domain, and Proposition 2.5 shows that $K$ is a (**)-extension of $\bar{D}$ in this case if and only if $\bar{D}$ is a semilocal PID. From this observation, the following corollary follows easily.

Corollary 2.6. Assume that $D$ is a Noetherian domain with quotient field $K \neq D$. The following conditions are equivalent.

(1) $D$ is a one-dimensional semilocal domain.

(2) $K$ is a simple ring extension of $D$.

(3) $K$ is $a\left(^{* *}\right)$-extension of $D$.

Corollary 2.6 presages both Theorem 4.7 and Theorem 5.1, which show, respectively, that a domain that is a (**)-extension of a Noetherian subring $D$ is finitely generated over $D$, and that an arbitrary (*)-extension of a Noetherian ring $R$ is finitely generated over $R$.

If $D$ is a Bezout domain, then $D_{M}$ is a valuation ring for each maximal ideal $M$ of $D$. Hence, the next result represents a deeper analysis of Proposition 2.5. The hypothesis that $V$ contains no minimal prime is included in the statement of Proposition 2.7 because, as is well known, $K$ is finitely generated over $V$ if and only if $V$ has a minimal prime ideal.

Proposition 2.7. Let $V$ be a valuation ring with quotient field $K$, and assume that $V$ has no minimal prime ideal. The following conditions are equivalent.

(1) $K$ is $a\left(^{*}\right)$-extension of $V$.

(2) $K$ is $a\left(^{* *}\right)$-extension of $V$.

(3) $K$ is not countably generated over $V$.

(4) If $\left\{P_{i}\right\}_{i=1}^{\infty}$ is a sequence of nonzero prime ideals of $V$, then $\cap_{i=1}^{\infty} P_{i} \neq(0)$.

Proof. The known ideal theory of valuation rings implies that (3) and (4) are equivalent, and (2) implies (3) since (2) and the negation of (3) imply that $K$ is finitely generated over $V$, contrary to the hypothesis that $V$ has no minimal prime ideal. Thus, we prove that (4) implies (1). Let $\left\{x_{i}\right\}_{i=1}^{\infty}$ be a countable subset of $K-V$ so that $y_{i}=x_{i}^{-1} \in V$ for each $i$. Since $V y_{i} V$ is a nonminimal prime ideal of $V$, then $y_{i} V$ contains a nonzero prime $P_{i}$ of $V$. Let $y$ be a nonzero element of $\cap_{i=1}^{\infty} P_{i}$. Since $y \in y_{i}^{k} V$ for each $i$ and each positive integer $k$, it follows that each $x_{i}^{k}$ belongs to $V y^{-1}$, whence $V\left[\left\{x_{i}\right\}_{1}^{\infty}\right] \subseteq V\left[y^{-1}\right]$ and $K$ is a $\left(^{*}\right)$-extension of $V$, as asserted.

An arbitrary well-ordered set is, to within isomorphism, the set of nonzero proper prime ideals of a valuation ring, ordered under $P_{\alpha}<P_{\beta}$ if $P_{\alpha} \supseteq P_{\beta}$. In particular, there exists a valuation ring $W$ with no minimal prime ideal such that no 
countable set of nonzero prime ideals of $W$ has intersection (0) (see, for example, [5, p. 1139]). For such a valuation ring $W$, it follows from Proposition 2.7 that the quotient field of $W$ is a $\left(^{*}\right)$-extension of $W$, but is not finitely generated over $W$.

We remark that there is a natural extension of Proposition 2.7 to the case of an arbitrary overring of a valuation ring. This result is stated without proof in Proposition 2.8.

Proposition 2.8. Let $W$ be a proper overring of the valuation ring $V$, let $P$ be the center of $W$ on $V$, and let $\mathcal{S}$ be the set of prime ideals of $V$ that properly contain $P$. The following conditions are equivalent.

(1) $W$ is $a\left(^{*}\right)$-extension of $V$.

(2) $W$ is $a\left({ }^{* *}\right)$-extension of $V$.

(3) $W$ is either finitely generated, or not countably generated, over $V$.

(4) Either (a) $\mathcal{S}$ contains a minimal element, or (b) $\mathcal{S}$ contains no minimal element and no countable subset of $\mathcal{S}$ has $P$ as its intersection.

As previously remarked, we give in $\$ 4$ an example of a $\left(^{* *}\right)$-overring $J$ of a domain $D$ such that $J$ is not a $\left(^{*}\right)$-overring of $D$.

3. $\left(^{* *}\right)$-extension. Much of this section is concerned with the problem of determining conditions on an integral domain $D$ in order that each ( $\left.{ }^{* *}\right)$-extension domain of $D$ is finitely generated over $D$. In Theorem 3.7, we prove that sufficient conditions for $D$ to have this property are that $D$ has Noetherian spectrum, each ideal of $D$ is countably generated, and $D$ satisfies d.c.c. for prime ideals. In Theorem 3.10 we show that a $\left({ }^{* *}\right)$-extension $T$ of a Noetherian ring $R$ is finitely generated over $R$ if and only if $T$ is Noetherian.

Theorem 3.1. Assume that $K$ is a subfield of the field $L$ and that $L / K$ is not a finitely generated field extension. Then there exists an infinite strictly ascending sequence $K<K_{1}<K_{2}<\cdots$ of intermediate fields such that $L=\cup_{i=1}^{\infty} K_{i}$.

Proof. We consider separately the cases where the transcendence degree of $L / K$ is finite or infinite. If the transcendence degree of $L / K$ is infinite, then by possible passage to a purely transcendental extension of $K$, we assume without loss of generality that a transcendence basis $B=\{x\}_{i=1}^{\infty}$ of $L / K$ is countably infinite. Let $K_{1}$ be the algebraic closure of $K\left(x_{1}\right)$ in $L$, let $K_{2}$ be the algebraic closure of $K_{1}\left(x_{2}\right)$ in $L$, etc. It follows from the fact that $B$ is a transcendence basis for $L / K$ that $L=\cup_{i=1}^{\infty} K_{i}$ and that $K_{i}<K_{i+1}$ for each $i$. Thus, the proof is complete in the case where tr.d. $L / K=\infty$.

If tr.d. $L / K$ is finite, then by adjoining a transcendence basis of $L / K$ to $K$, we may assume that $L / K$ is algebraic, but not finite dimensional. Choose a sequence $\left\{x_{i}\right\}_{i=1}^{\infty}$ in $L$ such that the sequence $K<K\left(x_{1}\right)<K\left(x_{1}, x_{2}\right)<\cdots$ strictly ascends. By Zorn's Lemma, there exists a subfield $K_{1}$ of $L$ containing $K$ such that $K_{1}$ is maximal with respect to the property that the sequence $K_{1} \subseteq K_{1}\left(x_{1}\right) \subseteq$ $K_{1}\left(x_{1}, x_{2}\right) \subseteq \cdots$ strictly ascends. Using Zorn's Lemma again, there exists a subfield $K_{2}$ of $L$ containing $K_{1}\left(x_{1}\right)$ such that $K_{2}$ is maximal with respect to the property that the sequence $K_{2} \subseteq K_{2}\left(x_{2}\right) \subseteq K_{2}\left(x_{2}, x_{3}\right) \subseteq \cdots$ strictly ascends. By 
induction, we obtain an ascending sequence $K_{1}<K_{2}<\cdots<K_{n}<\cdots$, where, for each $n, K_{n+1}$ is a subfield of $L$ containing $K_{n}\left(x_{n}\right)$ such that $K_{n+1}$ is maximal with respect to the property that the sequence $K_{n+1} \subseteq K_{n+1}\left(x_{n+1}\right) \subseteq$ $K_{n+1}\left(x_{n+1}, x_{n+2}\right) \subseteq \cdots$ strictly ascends. Let $F=\cup_{i=1}^{\infty} K_{i}$. We prove that $F=L$. Let $\theta \in L$ and let $f(Y)$ be the minimal polynomial for $\theta$ over $F$. For some $n$, $f(Y) \in K_{n}[Y]$, so $F$ and $K_{n}(\theta)$ are linearly disjoint over $K_{n}$. Therefore $K_{n}(\theta)<$ $K_{n}(\theta)\left(x_{n}\right)<K_{n}(\theta)\left(x_{n}, x_{n+1}\right)<\cdots$. Maximality of $K_{n}$ then implies that $\theta \in K_{n}$, so that $L=\cup_{n=1}^{\infty} K_{n}$, as asserted. This completes the proof of Theorem 3.1.

Corollary 3.2. Assume that the integral domain $D_{2}$ is a (**)-extension of its subring $D_{1}$. If $K_{i}$ is the quotient field of $D_{i}$, then $K_{2} / K_{1}$ is a finitely generated field extension.

Proof. If $K_{2} / K_{1}$ is not finitely generated, then $K_{2}$ is expressible as $\cup_{n=1}^{\infty} F_{n}$, where $\left\{F_{n}\right\}_{1}^{\infty}$ is a strictly ascending sequence of intermediate fields. Then $D_{2}=$ $\cup_{1}^{\infty}\left(D_{2} \cap F_{n}\right)$, where $D_{1} \subseteq D_{2} \cap F_{1}$ and $D_{2} \neq D_{2} \cap F_{n}$ for each $n$. This contradicts the hypothesis that $D_{2}$ is a $\left(^{* *}\right)$-extension of $D_{1}$, and therefore establishes the corollary.

We could give a much shorter proof of Theorem 3.1 if the following question (Q) had an affirmative answer.

(Q) Assume that $K$ is a subfield of the field $L$ and that $L / K$ is not finitely generated. Does there exist an intermediate field $F$ such that $L / F$ is countably generated, but not finitely generated?

This question seems to be open, however, since an affirmative answer to (Q) would imply that the following conjecture, stated by Bialynicki-Birula [3] (see also [7]), is correct.

CONJECTURE. Any nonprime field contains a proper subfield of countable (finite or infinite) codimension.

While Theorem 3.1 deals with the case where $L / K$ is not finitely generated as a field extension, the $\left(^{* *}\right)$-condition is stated in terms of ring generators. Proposition 3.3 addresses this discrepancy. Except for the statement in Proposition 3.3 that the domains $D_{i}$ are integrally closed, Proposition 3.3 is implied by Theorem 3.7.

Proposition 3.3. Assume that $K$ is a subfield of the field $L$ and that $L$ is not finitely generated as a ring extension of $K$. Then there exists a strictly ascending sequence $K<D_{1}<D_{2}<\cdots$ of integrally closed subrings of $L$ such that $L=$ $\cup_{i=1}^{\infty} D_{i}$.

Proof. If $L / K$ is not finitely generated as a field extension, then Proposition 3.3 follows from Theorem 3.1. Thus, assume that $L=K\left(\theta_{1}, \ldots, \theta_{n}\right)$ is finitely generated over $K$ where, necessarily, some $\theta_{i}$ is transcendental over $K$. Without loss of generality, we assume that $\theta_{1}$ is transcendental over $K$ and that $L$ is algebraic over $K\left[\theta_{1}\right]$. As $K\left[\theta_{1}\right]$ is a principal ideal domain with infinitely many maximal ideals, it follows from Corollary 2.6 that $K\left(\theta_{1}\right)$ is the union of a strictly ascending sequence $\left\{J_{i}\right\}_{i=1}^{\infty}$ of (necessarily integrally closed) overrings of $K\left[\theta_{1}\right]$. If $D_{i}$ is the integral closure of $J_{i}$ in $L$, then it follows that $K<D_{1}<D_{2}<\cdots<D_{n}<\cdots$ and $L=\cup_{1}^{\infty} D_{i}$, where each $D_{i}$ is integrally closed. 
Theorem 3.7 introduces a class of domains $D$ such that each $\left({ }^{* *}\right)$-extension domain of $D$ is finitely generated. The statement and proof of Theorem 3.7 requires some new terminology and two preliminary results. Let $M$ be a unitary module over the commutative ring $R$ with identity. We say that $M$ is $\omega_{0}$-Noetherian if each submodule of $M$ is countably generated; $R$ is an $\omega_{0}$-Noetherian ring if each ideal of $R$ is countably generated.

Proposition 3.4. A countably generated unitary module over an $\omega_{0}$-Noetherian ring is an $\omega_{0}$-Noetherian module.

Proof. Let $M=\sum_{1}^{\infty} R x_{i}$ be a countably generated unitary module over the $\omega_{0}$-Noetherian ring $R$ and $N$ be a submodule of $M$. Since

$$
N=\bigcup_{n=1}^{\infty}\left(N \cap \sum_{i=1}^{n} R x_{i}\right)
$$

it suffices to prove that each submodule $N \cap \sum_{i=1}^{n} R x_{i}$ is countably generated; to do so, we use induction on $n$. For $n=1, N \cap R x_{1}$ is $R$-isomorphic to an ideal of $R$ containing $\operatorname{Ann}\left(x_{1}\right)$, and hence $N \cap R x_{1}$ is countably generated since $R$ is $\omega_{0}^{-}$ Noetherian. If $N \cap\left(\sum_{1}^{k} R x_{i}\right)$ is countably generated, then to prove that $N^{\prime}=N \cap$ $\left(\sum_{1}^{k+1} R x_{i}\right)$ is countably generated, we need only show that $N^{\prime} /\left(N^{\prime} \cap \Sigma_{1}^{k} R x_{i}\right)$ is countably generated. This follows since

$$
N^{\prime} /\left(N^{\prime} \cap \sum_{1}^{k} R x_{i}\right) \simeq\left(N^{\prime}+\sum_{1}^{k} R x_{i}\right) / \sum_{1}^{k} R x_{i},
$$

a cyclic $R$-module.

Proposition 3.5. Consider the following conditions on a ring: (1) Noetherian spectrum, (2) d.c.c. for prime ideals, and (3) $\omega_{0}$-Noetherian. If $R$ is a ring satisfying one of these three conditions, then each finitely generated unitary extension ring of $R$ satisfies the same condition.

Proof. Let $S=R\left[s_{1}, \ldots, s_{n}\right]$ be a finitely generated unitary extension ring of $R$. If $R$ has Noetherian spectrum, then $S$ has Noetherian spectrum by Corollary 2.6 of $[10]$.

Assume that $R$ is $\omega_{0}$-Noetherian. Since $S$ is a countably generated $R$-module, then $S$ is an $\omega_{0}$-Noetherian $R$-module, hence an $\omega_{0}$-Noetherian ring.

Finally, assume that $R$ satisfies d.c.c. for prime ideals. To prove that $S$ satisfies the same condition, it is sufficient to prove this for the polynomial ring $R[X]$ in one variable over $R$; there it follows from the known result that if $P_{1}<P_{2}<P_{3}$ is a chain of three prime ideals of $R[X]$, then $P_{1} \cap R<P_{3} \cap R[4,(30.1)]$.

We state next a result in the same vein as Proposition 3.5. Proposition 3.6 is not used in the proof of Theorem 3.7, and we do not include the routine verification of its validity.

Proposition 3.6. Let $R$ be a ring with only finitely many maximal ideals $M_{1}, \ldots, M_{n}$. If $\pi$ is one of the conditions (1), (2), or (3) of Proposition 3.5, then $\pi$ is satisfied in $R$ if and only if $\pi$ is satisfied in each $R_{M_{i}}$. 
THEOREM 3.7. Assume that the integral domain $J$ is a $\left(^{* *}\right)$-extension of its subring D. If $D$ is $\omega_{0}$-Noetherian, has Noetherian spectrum, and satisfies d.c.c. on prime ideals, then $J$ is a finitely generated ring extension of $D$.

Proof. Let $L$ and $K$ denote the quotient fields of $J$ and $D$, respectively. By Corollary $3.2, L / K$ is a finitely generated field extension. Thus, there exists a finite subset $\left\{\theta_{i}\right\}^{n}$ of $J$ so that $L=K\left(\theta_{1}, \ldots, \theta_{n}\right)$. As $D_{1}=D\left[\theta_{1}, \ldots, \theta_{n}\right]$ satisfies the same three conditions that $D$ satisfies by Proposition 3.5 , and since $J$ is a (**)-extension of $D_{1}$, it suffices to prove Theorem 3.7 in the case where $J$ is an overring of $D$.

Let $\mathcal{S}$ be the set of prime ideals $P$ of $D$ such that $J \& D_{P}$. If $\mathcal{S}$ is empty, then $J=D$ and $J$ is finitely generated over $D$. Otherwise, $\delta$ has minimal elements since $D$ satisfies the d.c.c. for prime ideals; let $\mathcal{T}$ be the set of minimal elements of $\mathcal{S}$. We show first that $\mathcal{T}$ is finite. If not, then we choose a countably infinite subset $\left\{P_{i}\right\}_{i=1}^{\infty}$ of $\mathcal{T}$, and for each positive integer $n$, set $D_{n}=\cap_{i=n}^{\infty} D_{P_{i}}$. Then $D \subseteq D_{1} \subseteq$ $D_{2} \subseteq \cdots$ and $D \subseteq D_{1} \cap J \subseteq D_{2} \cap J \subseteq \cdots \subseteq J$. Note that $J \cap D_{n} \subset J$ for each $n$ since $D_{n} \subseteq D_{P_{n}}$. Finally, we show that $J=\cup_{i=1}^{\infty}\left(J \cap D_{i}\right)$-that is, that $J \subseteq$ $\cup_{i=1}^{\infty} D_{i}$. Let $t \in J$ and let $A_{t}=\{d \in D \mid d t \in D\}$ be the conductor of $t$ to $D$. We show that $t \notin D_{P_{i}}$ implies that $P_{i}$ is a minimal prime of $A_{t}$; since $A_{t}$ has only finitely many minimal primes ( $D$ has Noetherian spectrum), it will then follow that $t$ belongs to all but a finite number of the localizations $D_{P_{i}}$, and hence $t \in D_{n}$ for some $n$. Now $t \notin D_{P_{i}}$ implies that $A_{t} \subseteq P_{i}$, whence $P_{i}$ contains a minimal prime $P$ of $A_{t}$. Since $A_{t} \subseteq P$ implies that $t \notin D_{P}$, and hence that $J \in D_{P}$, we conclude from the choice of $P_{i} \in \mathcal{T}$ that $P_{i}=P$ is a minimal prime of $A_{t}$. This contradicts, however, the hypothesis that $J$ is a $\left(^{* *}\right)$-extension of $D$. Hence $\mathcal{T}$ is finite.

Let $\mathcal{T}=\left\{P_{1}, \ldots, P_{s}\right\}$, and choose a nonzero element $x$ of $\cap_{i=1}^{s} P_{i}$. The quotient ring $D[1 / x]$ of $D$ is the intersection of all localizations $D_{Q}$ of $D$ such that $Q$ is prime in $D$ and $x \notin Q$. Note that $J \subseteq D_{Q}$ for each such $Q$, for if not, then $Q$ contains some $P_{i}$, and hence $x \in Q$. Consequently, $J \subseteq D[1 / x]$. By Proposition $3.4, J$ is a countably generated $D$-module, hence a countably generated ring extension of $D$. Because $J$ is a $\left(^{* *}\right)$-extension of $D$, it follows that $J$ is a finitely generated ring extension of $D$, and this completes the proof of Theorem 3.7.

The class $\Re$ of domains satisfying conditions (1)-(3) of Proposition 3.5 includes the classes of Noetherian rings and finite-dimensional valuation rings [8, Corollary 11], and hence, by Proposition 3.6, the class of finite-dimensional semi-quasi-local Bezout domains. Thus, $\mathscr{T}$ is reminiscent of the class $\mathscr{F}$ considered in $[1, \S 4]$ consisting of rings over which each $\left(^{* *}\right)$-module is finitely generated. In $\$ 6$ we show (Theorem 6.1) that, in fact, $\mathcal{\Re}$ is a subclass of $\mathscr{F}$. At this point we note that while each $W^{*}$-domain is in $\mathscr{F}\left[1\right.$, Theorem 4.7], a $W^{*}$-domain need not be $\omega_{0}$ Noetherian; we correct this omission in Theoem 6.1 by providing an alternate to the $\omega_{0}$-Noetherian condition in its statement. In this connection, it would be interesting to determine if each $\left(^{* *}\right)$-extension domain of a $W^{*}$-domain $D$ is finitely generated over $D$.

Sharply in contrast with the case of $\left({ }^{* *}\right)$-extension domains, we give an example in $\S 4$ of a (**)-extension ring $R$ of a field $k$ such that $R$ is not finitely generated 
over $k$. In the remainder of the section we show, however, that there are some positive results in this direction if suitable restrictions are placed on the extension ring.

Proposition 3.8. Assume that $T$ is a unitary ring extension of the Noetherian ring $R$, that $T$ is reduced with only finitely many minimal prime ideals $P_{1}, P_{2}, \ldots, P_{n}$, and that $T / P_{i}$ is finitely generated over $R /\left(P_{i} \cap R\right)$ for each $i$. Then $T$ is a finitely generated extension of $R$.

Proof. We imbed $T$ in $S=\left(T / P_{1}\right) \oplus \cdots \oplus\left(T / P_{n}\right)$, noting that $S$ is a finitely generated $T$-module. The hypothesis implies that $S$ is also a finitely generated ring extension of $R$. A theorem of Artin and Tate [2, Theorem 1] then implies that $T$ is a finitely generated ring extension of $R$.

Proposition 3.9. Assume that $S$ is a unitary extension of the ring $R$, that $T$ is a finitely generated ring extension of $R$ in $S$, and that $S=T+N$, where $N$ is a finitely generated nilpotent ideal of $S$. Then $S$ is a finitely generated ring extension of $R$.

Proof. Assume that $N=\left(a_{1}, \ldots, a_{n}\right)$, and let $S_{1}=T\left[a_{1}, \ldots, a_{n}\right]$. We prove that $S=S_{1}$. Since $S=S_{1}+N$ and since $N$ is nilpotent, it suffices to prove that $S=S_{1}+N^{k}$ implies that $S=S_{1}+N^{k+1}$. Take $x \in N^{k}, x=\sum_{i=1}^{h} s_{i} m_{i}$, where $s_{i} \in S$ and $\left\{m_{i}\right\}_{1}^{h}$ is the set of monomials in $a_{1}, a_{2}, \ldots, a_{n}$ of degree $k$. We write each $s_{i}$ in the form $t_{i}+n_{i}$, where $t_{i} \in T$ and $n_{i} \in N$; thus $x=\sum_{1}^{h} t_{i} m_{i}+\sum_{1}^{h} n_{i} m_{i} \in$ $S_{1}+N^{k+1}$. It follows that $N^{k} \subseteq S_{1}+N^{k+1}$, so $S=S_{1}+N^{k+1}$, as was to be proved.

Theorem 3.10. Assume that $T$ is a $\left({ }^{* *}\right)$-extension of the Noetherian ring $R$. Then $T$ is finitely generated over $R$ if and only if $T$ is Noetherian.

Proof. The Hilbert Basis Theorem implies that $T$ is Noetherian if $T$ is finitely generated over $R$. Conversely, assume that $T$ is Noetherian and let $\left\{P_{i}\right\}_{i=1}^{n}$ be the set of minimal primes of $T$. By Proposition 2.2, $T / P_{i}$ is a (**)-extension of $R /\left(P_{i} \cap R\right)$ for each $i$, and hence $T /\left(\cap_{i=1}^{n} P_{i}\right)$ is a finite ring extension of $R /\left(\cap_{1}^{n}\left(P_{i} \cap R\right)\right)$ by Proposition 3.8. As $\cap_{1}^{n} P_{i}$ is a finitely generated nilpotent ideal of $T$, we then conclude from Proposition 3.9 that $T$ is finitely generated over $\boldsymbol{R}$.

As stated previously, the next section of the paper is devoted to the presentation of an example showing that even for a field $R$, a ( $\left.{ }^{* *}\right)$-extension of $R$ need not be finitely generated over $R$.

An analogue of Theorem 3.10 is valid if the condition that $R$ is Noetherian is weakened to the assumption that $R$ satisfies conditions (1)-(3) of Proposition 3.5; this is the content of the next result.

Theorem 3.11. Assume that the ring $R$ has Noetherian spectrum, is $\omega_{0}$-Noetherian, and satisfies d.c.c. for prime ideals. Let $T$ be a $\left({ }^{* *}\right)$-extension of $R$. Then $T$ is finitely generated over $R$ if either of the following conditions is satisfied.

(i) $T$ is a reduced ring with only finitely many minimal prime ideals.

(ii) $T$ is $\omega_{0}$-Noetherian and has only finitely many minimal primes. 
Proof. To prove (i), let $\left\{P_{i}\right\}_{i=1}^{n}$ be the set of minimal primes of $T$. For each $i$, $T / P_{i}$ is a (**)-extension of $R /\left(P_{i} \cap R\right)$. By Theorem 3.7, $T / P_{i}$ is finitely generated over $R /\left(P_{i} \cap R\right)$. Then as in the proof of Proposition 3.8, it follows that $T$ can be imbedded in $S$, where $S$ is a finitely generated ring extension of $R$ and a finite module extension of $T$. Since $R$ is $\omega_{0}$-Noetherian, $S$ is an $\omega_{0}$-Noetherian $R$-module. In particular, $T$ is a countably generated ring extension of $R$, and hence a finitely generated extension of $R$ since $T$ is a ( $\left.{ }^{* *}\right)$-extension of $R$. This establishes (i).

To prove (ii), let $B$ be the nilradical of $T$. By (i), it follows that $T / B$ is finitely generated over $R /(B \cap R)$. Thus, by a possible finite ring extension of $R$, we can assume without loss of generality that $T=R+B$. It is then clear that $T$ has Noetherian spectrum and satisfies d.c.c. for prime ideals. Consider first the case where $R$ is reduced. In this case, we show that $B$ is a $\left(^{* *}\right)$-ideal of $T$. Let $\left\{B_{i}\right\}_{i=1}^{\infty}$ be an ascending sequence of ideals of $T$ with union $B$. Then $T$ is the union of its chain $\left\{R+B_{i}\right\}_{i=1}^{\infty}$ of subrings. Therefore $T=R+B_{i}$ for some $i$, and since $R$ is reduced, $B=B_{i}$. Consequently, $B$ is a $\left(^{* *}\right)$-ideal, and Theorem 6.1 of $\S 6$ shows that $B$ is finitely generated. By Proposition 3.9, it follows that $T$ is finitely generated over $R$ in the case where $R$ is reduced. In the general case (we still assume that $T=R+B)$, the preceding case shows that $T /(B \cap R) T$ is finitely generated over $R /(B \cap R)$, hence $T=S+(B \cap R) T$ for some finitely generated ring extension $S$ of $R$. Because $B \cap R$ is a countably generated nil ideal of $R, B \cap R$ can be expressed as the union of an ascending sequence $\left\{C_{i}\right\}_{i=1}^{\infty}$ of nilpotent ideals of $R$. Since $T=\cup_{1}^{\infty}\left(S+C_{i} T\right)$ and $T$ is a $\left(^{* *}\right)$-extension of $R$, it follows that $T=S+$ $C_{i} T$ for some $i$, whence $T=S+C_{i}^{m} T$ for each $M$, and $T=S$ since $C_{i}$ is nilpotent. Therefore $T$ is finitely generated over $R$, and this completes the proof of Theorem 3.11 .

4. Some examples. Let $k$ be a field and let $N$ be an infinite set. We denote by $k^{N}$ the direct product of $k$ with itself over the index set $N$, and we denote by $k^{*}$ the diagonal imbedding of $k$ in $k^{N}$. Since the ring $k^{N}$ is not Noetherian, it is not a $\left(^{*}\right)$-extension of $k^{*}$ by Theorem 5.1. In this section, we show that $k^{N}$ is a $\left({ }^{* *}\right)$-extension of $k^{*}$ if and only if $k$ is a finite field. To simplify the notation, we write $S$ instead of $k^{N}$. Thus, we think of $S$ alternately as either the set of all functions from $N$ into $k$, under pointwise addition and multiplication, or as sequences $\left\{x_{i}\right\}_{i \in N}$ over $R$ indexed by $N$, where addition and multiplication are coordinatewise. For $f \in S$, we denote by $\mathcal{H}(f)$ the support of $f$-that is, $\mathcal{H}(f)=$ $\{n \in N \mid f(n) \neq 0\}$; the support of $\left\{x_{n}\right\}_{n \in N}$ is similarly defined as $\left\{i \in N \mid x_{i} \neq 0\right\}$. As is well known, the ring $S$ is absolutely flat (or von Neumann regular), meaning that each ideal of $S$ is idempotent or, alternatively, that $S_{p}$ is a field for each proper prime ideal $P$ of $S$. A significant difference between the cases $k$ finite or $k$ infinite is indicated by the next result.

Proposition 4.1. If $k$ is a finite field with $q$ elements, then $x^{q}=x$ for each $x \in S$ so that $S$ is integral over $k^{*}$. If $k$ is infinite, then $S$ is not algebraic over $k^{*}$.

Proof. The first statement is clear. If $k$ is infinite, choose a sequence $s=\left\{s_{i}\right\}_{1}^{\infty}$ $\in S$ with infinitely many distinct coordinates. If $f^{*}(X)$ is a nonzero polynomial 
over $k^{*}$, then $f^{*}(X)$ corresponds to a unique diagonal element $\{f(X)\}$ of $k[X]^{N}$. Moreover, $f^{*}(s)=\left\{f\left(s_{i}\right)\right\}_{i=1}^{\infty}$ is nonzero since $f(X)$ has only finitely many roots in $k$; thus, $s$ is not algebraic over $k^{*}$.

Corollary 4.2. Assume that the field $k$ is finite. Let $R$ be an $S$-overring of $k^{*}$ and let $M$ be a maximal ideal of $R$. Then $R$ is absolutely flat, $R=k^{*}+M$, and $R / M \simeq k$.

Proof. Assume that $|k|=q$. That $R$ is absolutely flat follows from the fact that $x^{q}=x$ for each $x \in R$. Also, the field $R / M$ is such that each of its elements satisfies the equation $x^{q}=x$. Therefore $R / M$ is finite with at most $q$ elements. Since $k^{*} \subseteq R$ and $k^{*} \cap M=(0)$, it then follows that $R=k^{*}+M$ and $R / M \simeq k$.

Proposition 4.3. If $k$ is infinite, then $S$ is not a $\left({ }^{* *}\right)$-extension of $k^{*}$.

Proof. Choose $s \in S$ so that $s$ is not algebraic over $k^{*}$. Then $G=\{f(s) \mid f(X)$ is a nonzero element of $\left.k^{*}[X]\right\}$ is a multiplicative system in $S$ that does not contain 0 . Hence, there exists a prime ideal $P$ of $S$ that misses $G$. Thus, $s+P$ is an element of the field $S / P$ transcendental over $k^{*}$. By Proposition 3.3, $S / P$ is not a $\left({ }^{* *}\right)$-extension of $k^{*}$, whence $S$ is not a $\left({ }^{* *}\right)$-extension of its subring $k^{*}+P$. Finally, this implies that $S$ is not a $\left({ }^{* *}\right)$-extension of $k^{*}$.

We remark that the proof of Proposition 4.3 shows that, in general, if $R_{2}$ is a unitary extension of the commutative ring $R_{1}$ with identity and if $R_{2}$ is not integral over $R_{1}$, then there exists a prime ideal $P$ of $R_{2}$ such that $R_{2} / P$ is not integral over $R_{1} /\left(P \cap R_{1}\right)$. On the other hand, if $R_{2} / M$ is integral over $R_{1} /\left(M \cap R_{1}\right)$ for each maximal ideal $M$ of $R_{2}$, then $R_{2}$ need not be integral over $R_{1}$. For example, $F[X] / M$ is integral over $F$ for each maximal ideal $M$ of the polynomial ring $F[X]$ over the field $F$.

As expected, the proof in Theorem 4.7 that $S$ is a $\left({ }^{* *}\right)$-extension of $k^{*}$, for $k$ finite, is more difficult than the proof of Proposition 4.3. The proof of Theorem 4.7 uses several preliminary results.

Proposition 4.4. Assume that the field $k$ is finite and that $R_{1}$ and $R_{2}$ are $S$-overrings of $k^{*}$ such that $R_{1}<R_{2}$. Then there exist distinct maximal ideals $M_{1}, M_{2}$ of $R_{2}$ such that $M_{1} \cap R_{1}=M_{2} \cap R_{1}$.

Proof. Since $R_{2}$ is integral over $R_{1}$, each maximal ideal of $R_{1}$ is the contraction of a maximal ideal of $R_{2}$. Choose $s \in R_{2}-R_{1}$, and let $C$ be the conductor of $s$ to $R_{1}$. We show that each maximal ideal of $R_{1}$ containing $C$ is the contraction of more than one maximal ideal of $R_{2}$. To do so, we prove the contrapositive: if $M$ is maximal in $R_{1}$ and is the contraction of a unique maximal ideal of $R_{2}$, then $M \beth C$. The maximal ideals of $R_{2}$ that contract to $M$ on $R_{1}$ are those which contain $M R_{2}$. Since $R_{2}$ is absolutely flat, it follows that $M R_{2}$ is maximal in $R_{2}$. Let $H=R_{1}-M$. Then $\left(R_{1}\right)_{H} \simeq R_{1} / M \simeq k$ and $\left(R_{2}\right)_{H} \simeq R_{2} / M R_{2} \simeq k$ by Corollary 4.3. Since $\left(R_{1}\right)_{H}$ is naturally imbedded in $\left(R_{2}\right)_{H}$ and each of these sets is finite, we conclude that $\left(R_{1}\right)_{H}=\left(R_{2}\right)_{H}$. This equality is easily seen to imply, however, that for each $x \in R_{2}$, the conductor of $x$ to $R_{1}$ is not contained in $M$. In particular, $C \notin M$ and this completes the proof. 
For an arbitrary field $k$, it is proved in [1] that each maximal ideal $M$ of $S$ is a $\left(^{* *}\right)$-ideal-that is, $M$ is not the union of a strictly ascending sequence of ideals of $S$. This result motivates the hypothesis of Proposition 4.5.

Proposition 4.5. Let $R$ be an absolutely flat ring such that each maximal ideal of $R$ is a (**)-ideal. Assume that $A$ is an ideal of $R$ contained in infinitely many maximal ideals of $R$. Then $A$ is contained in uncountably many maximal ideals.

Proof. By passage to the ring $R / A$, it suffices to consider the case where $A=(0)$. Since (0) has infinitely many minimal primes, $R$ is not Noetherian. Let $M$ be a maximal ideal of $R$ that is not finitely generated, and assume that the set $\left\{M_{i}\right\}_{1}^{\infty}$ of maximal ideals of $R$ distinct from $M$ is countable. For each $i$, choose $x_{i} \in M-M_{i}$. Then $M=\vee\left(\left\{x_{i}\right\}_{1}^{\infty}\right)=\left(\left\{x_{i}\right\}_{1}^{\infty}\right)$ is a countably generated (**)-ideal which is not finitely generated, an impossibility.

The statement of the next result uses the following terminology. Let $\mathcal{S}=\left\{A_{\alpha}\right\}$ be a family of ideals of the ring $R$. An element $A$ in $\mathcal{S}$ is said to be isolated in $\mathcal{S}$ if $A$ is not contained in the union of the members of $\delta$ distinct from $A$. No restrictions on the cardinality of $k$ are needed in Proposition 4.6.

Proposition 4.6. Let $\mathcal{S}$ be a countably infinite set of maximal ideals of the ring $S=k^{N}$, and assume that each element of $\mathcal{S}$ is isolated in $\mathcal{S}$. If $\mathcal{S}=\left\{P_{i}\right\}_{i=1}^{\infty} \cup$ $\left\{Q_{i}\right\}_{i=1}^{\infty}$ is a partition of $\mathcal{S}$ into two infinite subsets, then $\left(\cap_{1}^{\infty} P_{i}\right)+\left(\cap_{1}^{\infty} Q_{i}\right)=S$.

Proof. For each $i$, we assume that $f_{i}$ is an element of $P_{i}$ that belongs to no other element of $\mathcal{S}$ and that $g_{i} \in Q_{i}$ belongs to no other element of $\mathcal{S}$. Without loss of generality, we assume that each $f_{i}$ and each $g_{i}$ is idempotent, so that regarding these elements as sequences over $k$, they are $(0,1)$-sequences. For $j \neq i$, we have $1-f_{i}$, $1-g_{i} \in P_{j} \cap Q_{j}$, and hence $f_{i}-g_{i}=\left(1-g_{i}\right)-\left(1-f_{i}\right) \in \cap_{j \neq i}\left(P_{j} \cap Q_{j}\right)$. Also, $f_{i}-g_{i}$ is in neither $P_{i}$ nor $Q_{i}$. Let $e_{i}=\left(f_{i}-g_{i}\right)^{2} ; e_{i}$ is a $(0,1)$-sequence with the property that $e_{i} \in \cap\left(P_{j} \cap Q_{j}\right), e_{i} \notin P_{i} \cup Q_{i}$. Consider the sequence $\left\{b_{i}\right\}_{i=1}^{\infty}$ defined as follows:

$$
b_{1}=f_{1} e_{1}, \quad b_{2}=\left(1-e_{1}\right) e_{2} f_{2}, \quad b_{3}=\left(1-e_{1}\right)\left(1-e_{2}\right) e_{3} f_{3}, \ldots
$$

We note first that each $b_{i}$ is a $(0,1)$-sequence, and that $\mathcal{H}\left(b_{i}\right)$, the support of $b_{i}$, is disjoint from $\mathcal{H}\left(b_{j}\right)$ for $i<j$ since $\mathcal{H}\left(b_{i}\right) \subseteq \mathcal{H}\left(e_{i}\right)$ and $\mathcal{H}\left(b_{j}\right) \subseteq \mathcal{H}\left(1-e_{i}\right)=N-$ $\mathcal{H}\left(e_{i}\right)$. Thus, it is meaningful to write the "infinite sum" $b=\sum_{i=1}^{\infty} b_{i}$; it denotes the $(0,1)$-sequence with support $\cup_{i=1}^{\infty} \mathcal{H}\left(b_{i}\right)$. Hence $b$ is idempotent, and we complete the proof by showing that $b \in \cap_{1}^{\infty} P_{i}$ while $b \notin \cup_{i=1}^{\infty} Q_{i}$ (so that $1-b \in$ $\cap_{i=1}^{\infty} Q_{i}$, and $\cap_{1}^{\infty} P_{i}$ and $\cap_{1}^{\infty} Q_{i}$ are comaximal, as asserted). To show that $b \in P_{i}$, note that $b_{1}, \ldots, b_{i-1} \in P_{i}$ since $e_{1}, \ldots, e_{i-1}$ are in $P_{i}$. Moreover, $b_{i} \in P_{i}$ since $f_{i} \in P_{i}$. Now

$$
\mathcal{H}\left(\sum_{i+1}^{\infty} b_{j}\right)=\bigcup_{i+1}^{\infty} \mathcal{H}\left(b_{j}\right) \subseteq \mathcal{H}\left(1-e_{i}\right),
$$

and hence $e_{i} \Sigma_{i+1} b_{i}=0$. Since $e_{i} \notin P_{i}$, it follows that $\sum_{i+1}^{\infty} b_{j} \in P_{i}$, and hence $b=b_{1}+\cdots+b_{i}+\sum_{i+1}^{\infty} b_{j} \in P_{i}$. The same arguments show that $b-b_{i} \in Q_{i}$. But $b_{i}=\left(1-e_{1}\right), \ldots,\left(1-e_{i-1}\right) e_{i} f_{i} \notin Q_{i}$ since none of the factors is in $Q_{i}$. Whence, $b \notin Q_{i}$ for each $i$ and this completes the proof of Proposition 4.6. 
If $R_{1}$ is a subring of $R_{2}$ and $P$ is a prime ideal of $R_{2}$, then we say that $P$ is unibranched over $R_{1}$ if $P$ is the unique prime of $R_{2}$ with contraction $P \cap R_{1}$ on $R_{1}$; this terminology is used in the proof of Theorem 4.7.

THEOREM 4.7..$^{3}$ If $k$ is a finite field, then $S=k^{N}$ is a $\left(^{* *}\right)$-extension of $k^{*}$.

Proof. Assume, to the contrary, that $S=\cup_{i=1}^{\infty} R_{i}$, where $\left\{R_{i}\right\}_{1}^{\infty}$ is a strictly ascending sequence of $S$-overrings of $k^{*}$. By Proposition 4.4, there exists a maximal ideal of $R_{1}$ that is the contraction of more than one maximal ideal of $S$. Let $P_{1}$ and $Q_{1}$ be distinct maximals of $S$ with the same contraction to $R_{1}$. We note that if $M$ is an arbitrary maximal ideal of $S$, then $M$ is generated by $M \cap R_{n}$ for some $n$ since $\left(M \cap R_{1}\right) \subseteq\left(M \cap R_{2}\right) \subseteq \ldots$ is an ascending sequence of ideals with union $M$, and $M$ is a ( $\left.{ }^{* *}\right)$-ideal. Thus, there exists $n \geqslant 2$ so that $P_{1}$ and $Q_{1}$ are unibranched over $R_{n}$. Hence, by replacing the sequence $\left\{R_{i}\right\}_{i=1}^{\infty}$ by the sequence $R_{1}<R_{n}<$ $R_{n+1}<\ldots$, we assume without loss of generality that $P_{1}$ and $Q_{1}$ are unibranched over $R_{2}$. We continue this process, obtaining distinct primes $P_{2}, Q_{2}$ of $S$ with the same contraction to $R_{2}$ such that $P_{2}$ and $Q_{2}$ are unibranched over $R_{3}$. By induction, we obtain an infinite set of pairs $P_{i}, Q_{i}$ of distinct primes of $S$ such that $P_{i}$ and $Q_{i}$ have the same contraction to $R_{i}$, but are unibranched over $R_{i+1}$ for all $i$. Note that there is no duplication in the listing $\left\{P_{1}, Q_{1}, P_{2}, Q_{2}, \ldots\right\}$, for if $i<j$, then $P_{i}$ and $Q_{i}$ are unibranched over $R_{j}$, while $P_{j}$ and $Q_{j}$ are not unibranched over $R_{j}$.

We propose now to obtain a subsequence $\left\{\left(P_{n_{i}}, Q_{n_{i}}\right)\right\}_{i=1}^{\infty}$ so that each element of the set $\mathcal{S}=\left\{P_{n_{i}}\right\}^{\infty} \cup\left\{Q_{n_{i}}\right\}_{1}^{\infty}$ is isolated in $\mathcal{S}$. This is done recursively, obtaining first a subsequence $\left\{\left(P_{a_{i}}, Q_{a_{i}}\right)\right\}_{i=1}^{\infty}$ of $\left\{\left(P_{i}, Q_{i}\right)\right\}_{1}^{\infty}$ so that $P_{a_{1}}$ and $Q_{a_{1}}$ are isolated in $\left\{P_{a_{1}}\right\}_{1}^{\infty} \cup\left\{Q_{a_{i}}\right\}_{1}^{\infty}$, as follows. The ideal $\cap_{1}^{\infty} P_{i}=A$ is contained in infinitely many maximal ideals of $S$, and hence in uncountably many maximal ideals of $S$ by Proposition 4.5. Choose a prime ideal $P$ of $S$, distinct from each $P_{i}$ and each $Q_{i}$, such that $\cap_{1}^{\infty} P_{i} \subseteq P$. Let $f_{1}$ be an idempotent element in $P_{1}-\left(P \cup Q_{1}\right)$. Then $\cap_{1}^{\infty} P_{i}$ can be expressed as $B \cap C$, where $B$ is the intersection of the primes $P_{i}$ that contain $f_{1}$ and $C$ is the intersection of those $P_{i}$ that contain $1-f_{i}$. Then $B C \subseteq P$ with $B \& P$ implies that $C \subseteq P$, and hence infinitely many of the primes $P_{i}$ contain $1-f_{1}$. Thus, we obtain a subsequence

$$
P_{1}, P_{s_{1}}, P_{s_{2}}, \ldots
$$

of

$$
P_{1}, P_{2}, P_{3}, \ldots \text {, }
$$

where $\left\{P_{s_{i}}\right\}_{1}^{\infty}$ is the family of ideals $P_{i}$ that contain $1-f_{1}$; without loss of generality, we assume that $1-f_{1} \in R_{s_{1}}$, so that $1-f_{1} \in P_{s_{t}} \cap R_{s_{t}}=Q_{s_{t}}$ for each $t \geqslant 1$. Hence, $P_{1}$ is isolated in the set

$$
\mathcal{T}=\left\{P_{1}\right\} \cup\left\{Q_{1}\right\} \cup\left\{P_{s_{i}}\right\}_{1}^{\infty} \cup\left\{Q_{s_{i}}\right\}_{1}^{\infty} \text {. }
$$

Now we basically repeat this process for $Q_{1}$ and the set $\mathcal{T}$; to wit we choose a prime ideal $Q$ of $S$ containing $Q_{1} \cap\left(\cap_{1}^{\infty} Q_{s_{i}}\right)$ such that $Q \notin \mathcal{T}$, and we choose an

\footnotetext{
${ }^{3}$ Koppelberg and Tits [8] show that if $\mathscr{P}(X)$ is the ring of subsets of the infinite set $X$, then $\mathscr{P}(X)$ is not the union of an infinite strictly ascending sequence of subrings. This statement is, of course, equivalent to the case of Theorem 4.7 where $k=\mathrm{GF}(2)$.
} 
idempotent $g_{1} \in Q_{1}-\left(P_{1} \cup Q\right)$. In this way we obtain a subsequence

$$
Q_{1}, Q_{t_{1}}, Q_{t_{2}}, \ldots
$$

of

$$
Q_{1}, Q_{s_{1}}, Q_{s_{2}}, \ldots
$$

such that $P_{1}$ and $Q_{1}$ are isolated in the set $\left\{P_{1}\right\} \cup\left\{Q_{1}\right\} \cup\left\{P_{t_{i}}\right\}_{1}^{\infty} \cup\left\{Q_{t_{i}}\right\}_{1}^{\infty}$. We let $n_{1}=1$. Assume that we have subsequences

$$
P_{n_{1}}, \ldots, P_{n_{m}}, P_{u_{1}}, P_{u_{2}}, \ldots
$$

and

$$
Q_{n_{1}}, \ldots, Q_{n_{m}}, Q_{u_{1}}, Q_{u_{2}}, \ldots
$$

of (1) and

$$
Q_{1}, Q_{2}, \ldots
$$

so that each $P_{n_{i}}$ and each $Q_{n_{i}}$ is isolated in

$$
\text { U }=\left\{P_{n_{i}}\right\}_{1}^{m} \cup\left\{P_{u_{i}}\right\}_{1}^{\infty} \cup\left\{Q_{n_{i}}\right\}_{1}^{m} \cup\left\{Q_{u_{i}}\right\}_{1}^{\infty} .
$$

Let $G$ be a prime ideal of $S$ containing $\left(\cap_{1}^{m} P_{n}\right) \cap\left(\cap_{1}^{\infty} P_{u_{i}}\right)$ such that $G \notin \mathcal{Q}$. Choosing an idempotent

$$
f_{m+1} \in P_{u_{1}}-\left(\left(\bigcup_{1}^{m}\left(P_{n_{i}} \cup Q_{n_{i}}\right)\right) \cup G \cup Q_{u_{1}}\right),
$$

we obtain, as in the case $m=1$, a subsequence

$$
P_{n_{1}}, \ldots, P_{n_{m}}, P_{u_{1}}, P_{v_{1}}, P_{v_{2}}, \ldots
$$

of (5) so that each $P_{n_{i}}$, each $Q_{n_{i}}$, and $P_{u_{1}}$ is isolated in the set

$$
\left\{P_{n_{i}}\right\}_{1}^{m} \cup\left\{Q_{n_{i}}\right\}_{1}^{m} \cup\left\{P_{u_{1}}\right\} \cup\left\{Q_{u_{1}}\right\} \cup\left\{P_{v_{i}}\right\}_{1}^{\infty} \cup\left\{Q_{v_{i}}\right\}_{1}^{\infty} \text {. }
$$

Because the notation is cumbersome, we omit the extension to a subsequence where $Q_{u_{1}}$ is also isolated. We then set $n_{m+1}=u_{1}$. By mathematical induction we obtain the desired subsequence $\left\{\left(P_{n_{i}}, Q_{n_{i}}\right)\right\}^{\infty}$ of $\left\{\left(P_{i}, Q_{i}\right)\right\}^{\infty}$. By Proposition 4.6, the ideals $\cap_{1}^{\infty} P_{n_{i}}$ and $\cap_{1}^{\infty} Q_{n_{i}}$ are comaximal. Choose an element $f \in \cap_{1}^{\infty} P_{n_{i}}$ such that $1-f \in \cap_{1}^{\infty} Q_{n_{i}}$. For some $t$ we have $f \in R_{n_{i}}$, whence $f \in P_{n_{i}} \cap R_{n_{i}}=Q_{n_{i}} \cap R_{n_{i}}$, and this contradicts the fact that $Q_{n_{i}}$ is a proper ideal of $S$. We conclude that $S$ is a $\left({ }^{* *}\right)$-extension of $k^{*}$.

It follows from Theorem 5.1 that a $\left(^{*}\right)$-extension of a Noetherian ring is a finitely generated extension. Since $k^{N}$ is obviously not finitely generated over $k^{*}$, Theorem 4.7 provides an example where $\left({ }^{* *}\right)$ does not imply $\left({ }^{*}\right)$; this example can be extended to that of an overring of an integral domain as follows. Let $k$ be a finite field; the ring $k^{N}$ is the homomorphic image of an appropriate polynomial ring $J$ over $k$. Let $D$ be the inverse image of $k^{*}$ under the homomorphism; thus $D=k+A$, where $A$ is the kernel of the homomorphism. Since $A \neq(0)$, the domain $J$ is an overring of $D$ (in fact, $J$ is the integral closure of $D$ ), and because of the corresponding properties for $k^{N}$ and $k^{*}$, it follows easily that $J$ is a $\left({ }^{* *}\right)$-overring of $D$, but not a $\left(^{*}\right)$-overring of $D$. Also in this connection, we remark that $D$ 
and $J$ provide an example of an integral $\left({ }^{* *}\right)$-ring extension that is not a $\left(^{* *}\right)$-module extension. It is easy to see that the converse is always true-that is, if a ring extension $S$ of $R$ is a $\left(^{* *}\right)-R$-module, then $S$ is also a (**)-ring extension of $R$.

5. (*)-extensions. Assume that $R$ is a ring satisfying conditions (1)-(3) of Proposition 3.5-that is, $R$ has Noetherian spectrum, is $\omega_{0}$-Noetherian, and satisfies d.c.c. for prime ideals. We prove in Theorem 5.3 that each $\left({ }^{*}\right)$-extension of $R$ is finitely generated over $R$. Because of the simplicity of its proof, we present the special case of Theorem 5.3 in which $R$ is Noetherian in Theorem 5.1. Results of $\$ 4$ show that analogues of these results for $\left({ }^{* *}\right)$-extensions fail miserably.

THEOREM 5.1. $A\left({ }^{*}\right)$-extension of a Noetherian ring is a finitely generated extension.

Proof. Let $T$ be a $\left({ }^{*}\right)$-extension of the Noetherian ring $R$. By Theorem 3.10, it suffices to prove that $T$ is Noetherian, and for this purpose, it is enough to show that each ideal of $T$ is a $\left(^{*}\right)$-ideal [1, Proposition 1.2]. Let $I$ be an ideal of $T$, let $\left\{t_{i}\right\}_{1}^{\infty}$ be a countable subset of $I$, and let $S$ be a finitely generated extension of $R$ in $T$ containing $R\left[\left\{t_{i}\right\}_{1}^{\infty}\right]$. Then $S$ Noetherian implies that $I \cap S$ is a finitely generated ideal of $S$ containing $\left\{t_{i}\right\}_{1}^{\infty}$. Thus, $(I \cap S) T$ is a finitely generated ideal of $T$ contained in $I$ and containing $\left\{t_{i}\right\}_{1}^{\infty}$. Hence $I$ is a $\left(^{*}\right)$-ideal, and this completes the proof of the theorem.

Proposition 5.2. Assume that $S$ is a unitary extension ring of the ring $R$. If $S$ has infinitely many minimal primes, then there exists a countable subset $\left\{s_{i}\right\}_{i=1}^{\infty}$ of $S$ such that the ring $R\left[\left\{s_{i}\right\}_{1}^{\infty}\right]$ has infinitely many minimal primes.

Proof. Choose a countably infinite set $\left\{P_{i}\right\}_{i=1}^{\infty}$ of minimal primes of $S$. Pick $x_{1} \in P_{1}$. Since $x_{1}$ belongs to a minimal prime of $S$, there exists $y_{1} \in S-P_{1}$ such that $x_{1} y_{1}$ is nilpotent. Next pick $x_{2} \in P_{2}-P_{1}$ and $y_{2} \in S-P_{2}$ such that $x_{2} y_{2}$ is nilpotent. Having chosen $x_{1}, y_{1}, \ldots, x_{n}, y_{n}$, choose $x_{n+1} \in P_{n+1}-$ $\left(P_{1} \cup \cdots \cup P_{n}\right)$ and $y_{n+1} \in S-P_{n+1}$ so that $x_{n+1} y_{n+1}$ is nilpotent. By induction, we can find an infinite such double sequence $\left\{x_{1}, y_{1}, x_{2}, y_{2}, \ldots\right\}$. Let $T=R\left[x_{1}, y_{1}, x_{2}, y_{2}, \ldots\right]$; we claim that $T$ has infinitely many minimal primes. For each $i$, let $Q_{i}$ be a minimal prime of $T$ contained in $P_{i} \cap T$. We show that $Q_{i} \neq Q_{j}$ if $i<j$. Thus $x_{j} y_{j}$ nilpotent implies $x_{j} y_{j} \in Q_{i} \cap Q_{j}$. Now $y_{j} \notin P_{j} \supseteq Q_{j}$ implies $x_{j} \in Q_{j}$ and $x_{j} \notin P_{i}$ implies $x_{j} \notin Q_{i} \subseteq P_{i}$. Therefore $Q_{i} \neq Q_{j}$ and $T$ has infinitely many prime ideals, as asserted.

TheOrem 5.3. Assume that $R$ is an $\omega_{0}$-Noetherian ring with Noetherian spectrum and with d.c.c. on prime ideals. If $T$ is a $\left(^{*}\right)$-extension of $R$, then $T$ is finitely generated over $R$.

Proof. We show first that $T$ has only finitely many minimal primes. If not, then Proposition 5.2 shows that there exists a countably generated extension $S$ of $R$ in $T$ such that $S$ has infinitely many minimal primes. Because $T$ is a $\left(^{*}\right)$-extension of $R$, there exists a finitely generated extension $S_{1}$ of $R$ in $T$ containing $S$. Then $S_{1}$ has Noetherian spectrum, and hence has only finitely many minimal primes. Since each minimal prime of $S$ is the contraction to $S$ of a minimal prime of $S_{1}$, it follows that 
$S$ has only finitely many minimal primes, a contradiction. Hence $T$ has only finitely many minimal primes, as asserted. Part (i) of Theorem 3.11 then shows that $T$ is finitely generated over $R$ if $T$ is reduced. In any case, if $B$ is the nilradical of $T$, then by a possible finite ring extension of $R$, we assume without loss of generality that $T=R+B$.

Assume first that $R$ is reduced. In this case, we prove that $B$ is a $\left(^{*}\right)$-module over $R$. Thus, let $\left\{b_{i}\right\}_{i=1}^{\infty}$ be a countable subset of $B$, and choose $t_{1}, \ldots, t_{k} \in T$ so that $R\left[\left\{b_{i}\right\}^{\infty}\right] \subseteq R\left[t_{1}, \ldots, t_{k}\right]$. Since $T=R+B$, we may assume that each $t_{i} \in B$. Because $B \cap R=(0)$, it then follows that each $b_{i}$ belongs to the $R$-module generated by all monomials in $\left\{t_{1}, \ldots, t_{k}\right\}$, a finite set since each $t_{i}$ is nilpotent. This proves that $B$ is a $\left({ }^{*}\right)$-module over $R$. Then Theorem 6.1 of the next section shows that $B$ is a finitely generated $R$-module, so $T$ is finitely generated in the case where $R$ is reduced. In the general case, the preceding case shows that $T=S+$ $(B \cap R) T$ for some finitely generated ring extension $S$ of $R$, and the remainder of the proof is identical to that of Theorem 3.11 from the same stage. This completes the proof of Theorem 5.3.

6. An expansion of the class $\mathscr{F}$. Define $\mathscr{T}$ to be the class of rings $R$ such that (1) $R$ has Noetherian spectrum, (2) d.c.c. for prime ideals is satisfied in $R$, and either (3) $R$ is $\omega_{0}$-Noetherian, or (4) each ideal of $R$ contains a power of its radical. Conditions (1)-(3) are the defining properties of the class $\Re$ of domains considered in $\S 3$. We prove in Theorem 6.1 that $\mathfrak{T}$ is a subclass of the class $\mathscr{F}$ of rings over which each $\left(^{* *}\right)$-module is finitely generated; this is the motivation for the addition of condition (4) in the definition of the class $\mathfrak{N}$-Theorem 4.7 of [1] shows that $\mathcal{F}$ contains the class of $W^{*}$-rings, and while a $W^{*}$-ring satisfies conditions (1), (2), and (4), such a ring need not be $\omega_{0}$-Noetherian. Thus, each of Theorems 4.2, 4.7, and 4.10 of [1] follows from Theorem 6.1. Note that the class $\Re$ contains rings that were not known to be in $\mathcal{F}$ from the results of [1]. There exist, for example, two-dimensional, countable, strongly Laskerian rings that are not Noetherian; such a ring $R$ satisfies (1)-(4), and hence is in $\Re$, but $R$ is not Noetherian, is not a finite-dimensional chained ring, and is not a $W^{*}$-ring.

THEOREM 6.1. $\left.A{ }^{(* *}\right)$-module over a ring in the class $\mathfrak{T}$ is finitely generated; that is $\mathfrak{T}$ is a subclass of the class $\mathscr{F}$.

Proof. Assume that $R \in \mathscr{T}$. We show first that $R / A \in \mathcal{F}$ for each radical ideal $A$ of $R$. If this is not the case, then since $R$ has Noetherian spectrum, there exists a radical ideal $B$ of $R$ maximal in the set of radical ideals $A$ of $R$ such that $R / A \notin \mathcal{F}$. Replacing $R$ by $R / B$, we can therefore assume that $R$ is a reduced ring with the property that there exists a nonfinitely generated (**)-module $M$ over $R$, whereas $R / C \in \mathcal{F}$ for each nonzero radical ideal $C$ of $R$. We propose to show that this assumption leads to a contradiction. Let $A$ be a nonzero proper ideal of $R$ and let $C=\vee A$. Either (3) or (4) implies that the ideal $C / A$ of $R / A$ is the union of an ascending sequence $A_{1} / A \subseteq A_{2} / A \subseteq \ldots$ of nilpotent ideals of $R / A$. Since $(R / A) /(C / A) \simeq R / C \in \mathcal{F}$, it follows from Lemma 4.3 of [1] that the ring $R / A$ is in $\mathscr{F}$. If $R$ is not an integral domain, the proposed contradiction is now easy to 
obtain: let $x$ and $y$ be nonzero elements of $R$ such that $x y=0$. Then $M / x M$ is a (**)-module over $R$ and over $R /(x)$ and hence is finitely generated over $R$ since $R /(x) \in \mathcal{F}$. Also, $x M$ is a $\left(^{* *}\right)$-module over $R$ and over $R /(y)$ [1, Lemma 4.8], hence a finite generated $R$-module by the same argument. Consequently, $M$ is a finitely generated $R$-module, contrary to assumption. This leads to consideration of the case where $R$ is an integral domain.

In the case where $R$ is an integral domain, we let $H=R-\{0\}$ and let $K=R_{H}$ be the quotient field of $R$. Then $M_{H}$ is a $\left(^{* *}\right)$-module over $K$, hence a finitely generated vector space over $K$. It follows that there exists a finitely generated free $R$-submodule $F$ of $M$ such that $M / F$ is a torsion $R$-module. As $M / F$ is a (**)-module over $R$ and our aim is to prove that $M$ is finitely generated, we assume without loss of generality that $M$ is a nonzero torsion module over $R$. Let $\Lambda$ be the set of primes $P$ of $R$ such that $\operatorname{Ann}(m) \subseteq P$ for some $m \in M$. Since $R$ satisfies d.c.c. for prime ideals, the set $\Lambda$ contains minimal elements; let $\left\{P_{\alpha}\right\}$ be the set of minimal elements of $\Lambda$. We consider separately the cases where $\left\{P_{\alpha}\right\}$ is finite or infinite. If $\left\{P_{\alpha}\right\}$ is finite, we choose a nonzero element $x \in \cap_{\alpha} P_{\alpha}$. For each $m \in M$, we have $x \in \vee \operatorname{Ann}(m)$, so $x^{n} m=0$ for some $n$. Let $M_{n}=\left\{m \in M \mid x^{n} m\right.$ $=0\}$. Then $M_{1} \subseteq M_{2} \subseteq \ldots$ and $M=\cup_{n=1}^{\infty} M_{n}$. Since $M$ is a (**)-module, we have $M=M_{n}$ for some $n$. Thus, $M$ is a $\left(^{* *}\right)$-module over $R /\left(x^{n}\right)$, hence finitely generated over $R /\left(x^{n}\right)$ and over $R$. This contradiction leads to the case where $\left\{P_{\alpha}\right\}$ is infinite. In this case, choose a countably infinite subset $\left\{\boldsymbol{P}_{i}\right\}_{i=1}^{\infty}$ of $\left\{\boldsymbol{P}_{\alpha}\right\}$, and for each positive integer $r$, define

$$
M_{r}=\left\{m \in M \mid \operatorname{Ann}(m) \& P_{i} \text { for any } i \geqslant r\right\} .
$$

It is clear that $M_{r}$ is a submodule of $M$ and that $M_{1} \subseteq M_{2} \subseteq \ldots$ We note that $M_{r} \neq M$ for each $r$, for there exists $m_{r} \in M$ such that $P_{r}$ is a minimal prime of $\operatorname{Ann}\left(m_{r}\right)$, and hence $m_{r} \in M-M_{r}$. Also we note that $M=\cup_{r=1}^{\infty} M_{r}$, for if $m \in M$, then $\operatorname{Ann}(m)$ has only finitely many minimal primes in $R$. Thus, the assumption that $\Lambda$ is infinite contradicts the fact that $M$ is a $\left(^{* *}\right)$-module, and this resolves the case where $R$ is an integral domain. Finally, we conclude that the assumption that $R / A \notin \mathscr{F}$ for some radical ideal $A$ of $R$ is false, so in particular, $R / V(0) \in \mathcal{F}$. Since either (3) or (4) implies that $\vee(0)$ is the union of an ascending sequence of nilpotent ideals of $R$, we then conclude as in a previous argument that $R \in \mathscr{F}$, and this completes the proof of Theorem 6.1.

In connection with Theorem 6.1 and the results in [1] concerning the class $\mathscr{F}$, it would be interesting to determine if any one-dimensional integral domain with Noetherian spectrum is in $\mathscr{F}$. We conclude this article with the following result showing that this is at least the case for integrally closed domains.

Proposition 6.2. If $R$ is a one-dimensional integrally closed domain with Noetherian spectrum, then $R$ is in $\mathcal{F}$.

Proof. We first show for any nonzero $x$ in $R$ that $R /(x)$ is in $\mathscr{F}$. Since $R$ is integrally closed, there exists a set $\left\{V_{\alpha}\right\}$ of valuation rings such that $R=\cap_{\alpha} V_{\alpha}$. Hence $(x)=\bigcap_{\alpha} x V_{\alpha}$. We observe that for each positive integer $n, A_{n}=$ $\left\{y \in R \mid y^{n} \in(x)\right\}$ is an ideal in $R$. For if $y, z \in A_{n}$, then $(y-z)^{n} \in x V_{\alpha}$ for each 
$V_{\alpha}$, so that $(y-z)^{n} \in x R$, and $y-z \in A_{n}$. Moreover, $A_{n}^{n} \subseteq(x)$. We have $\vee(x)$ $=\cup_{n=1}^{\infty} A_{n}=A$, and since $R$ is one-dimensional with Noetherian spectrum, $R / A$ is a finite product of fields and hence is in $\mathscr{F}$. By [1, Lemma 4.3], $R /(x)$ is in $\mathscr{F}$.

Let $M$ be a $\left({ }^{* *}\right)$-module over $R$. We wish to show that $M$ is finitely generated, and for this purpose, as in the proof of 6.1 , we may assume that $M$ is a torsion $R$-module. Moreover, again as in the proof of 6.1, the fact that $M$ is a $\left({ }^{* *}\right)$-module implies that the set $\Lambda$ of prime ideals $P$ of $R$ such that $\operatorname{Ann}(m) \subseteq P$ for some nonzero $m \in M$ is a finite set, say $\Lambda=\left\{P_{1}, \ldots, P_{s}\right\}$. Since $M$ is a torsion module, each $P_{i} \neq(0)$. Let $y$ be a nonzero element of $\cap_{i=1}^{s} P_{i}$. For each $m \in M$, we have $y \in \vee \operatorname{Ann}(m)$, so $y^{n} m=0$ for some $n$. Let $M_{n}=\left\{m \in M \mid y^{n} m=0\right\}$. Then $M_{1} \subseteq$ $M_{2} \subseteq \ldots$, and $M=M_{n}$ for some $n$. Thus, $M$ is a $\left.{ }^{(*}\right)$-module over $R /\left(y^{n}\right)$. Hence $M$ is finitely generated.

ACKNOWLEDGement. We would like to thank Leonard Lipschitz for his help in connection with the material in $\$ 4$. We are indebted to him for the proof of Theorem 4.7.

\section{REFERENCES}

1. J. T. Arnold, R. Gilmer and W. Heinzer, Some countability conditions in a commutative ring, Illinois J. Math. 21 (1977), 648-665.

2. E. Artin and J. T. Tate, A note on finite ring extensions, J. Math. Soc. Japan 3 (1951), 74-77.

3. A. Bialynicki-Birula, On subfields of countable codimension, Proc. Amer. Math. Soc. 35 (1972), 354-356.

4. R. Gilmer, Multiplicative ideal theory, Marcel Dekker, New York, 1972.

5. _ A note on the quotient field of the domain $D[[X]]$, Proc. Amer. Math. Soc. 18 (1967), $1138-1140$.

6. R. Gilmer and W. Heinzer, Cardinality of generating sets for ideals of a commutative ring, Indiana Univ. Math. J. 26 (1977), 791-798.

7. C. U. Jensen, Some remarks on valuations and subfields of given codimension in algebraically closed fields, Publ. Math. Debrecen 24 (1977), 317-321.

8. S. Koppelberg and J. Tits, Une propriété des produits directs infinis de groupes finis isomorphes, $\mathrm{C}$. R. Acad. Sci. Paris Ser. A 279 (1974), 583-585.

9. M. Nagata, Local rings, Interscience, New York, 1962.

10. J. Ohm and R. Pendleton, Rings with Noetherian spectrum, Duke Math. J. 35 (1968), 631-640.

Department of Mathematics, Florida State University, Tallahassee, Florida 32306

Department of Mathematics, Purdue University, West Lafayette, Indana 47907 